\title{
Numerical Simulation of Shoaling Internal Solitary Waves in Two-layer Fluid Flow
}

\author{
${ }^{1}$ M. H. Hooi, ${ }^{2}$ W. K. Tiong, ${ }^{3}$ K. G. Tay ${ }^{*},{ }^{4}$ K. L. Chiew and ${ }^{5}$ S. N. Sze \\ ${ }_{1,2,4,5}$ Faculty of Computer Science and Information Technology, \\ Universiti Malaysia Sarawak, 94300 Kota Samarahan, Sarawak, Malaysia \\ ${ }^{3}$ Department of Communication Engineering, Faculty of Electric and Electronic, \\ Universiti Tun Hussein Onn Malaysia. 86400 Parit Raja, Johor, Malaysia \\ ${ }^{*}$ Corresponding author: tay@uthm.edu.my
}

Article history

Received: 13 October 2017

Received in revised form: 4 May 2018

Accepted: 20 May 2018

Published on line: 1 December 2018

\begin{abstract}
In this paper, we look at the propagation of internal solitary waves over three different types of slowly varying region, i.e. a slowly increasing slope, a smooth bump and a parabolic mound in a two-layer fluid flow. The appropriate mathematical model for this problem is the variable-coefficient extended Korteweg-de Vries equation. The governing equation is then solved numerically using the method of lines. Our numerical simulations show that the internal solitary waves deforms adiabatically on the slowly increasing slope. At the same time, a trailing shelf is generated as the internal solitary wave propagates over the slope, which would then decompose into secondary solitary waves or a wavetrain. On the other hand, when internal solitary waves propagate over a smooth bump or a parabolic mound, a trailing shelf of negative polarity would be generated as the results of the interaction of the internal solitary wave with the decreasing slope of the bump or the parabolic mound. The secondary solitary waves is observed to be climbing the negative trailing shelf.
\end{abstract}

Keywords Internal solitary waves; extended KdV; the method of lines; two-layer fluid flow; shoaling solitary waves

Mathematics Subject Classification 35Q51, 35Q53, 65M20.

\section{Introduction}

It is widely accepted that the propagation of internal waves in shallow seas is based on the Korteweg-de Vries (KdV) equation. Benney [1] and Benjamin [2] were among the first who derived it in the context of internal waves followed by many other authors [3] - [6]. However, when the nonlinear coefficient of the $\mathrm{KdV}$ equation is close to zero, a cubic nonlinear term is needed [7] - [8]. Thus, the appropriate model for this situation is the extended KdV (eKdV) 
equation. The canonical form of the eKdV equation, more commonly known as the Gardner equation is given by

$$
u_{t}+6 \alpha u u_{x}+6 \beta u^{2} u_{x}+\gamma u_{x x x}=0 .
$$

One of the most common applications of the $\mathrm{eKdV}$ equation is to describe the propagation of the large-amplitude internal waves in stratified fluid flow (see [9] - [12] and references therein). The coefficient $\beta$ in (1) can be both positive and negative depending on the physical problems. In the context of internal waves, it depends on the stratification of the fluid. When $\beta=0$, then (1) becomes the well-known KdV equation

$$
u_{t}+6 \alpha u u_{x}+\gamma u_{x x x}=0 .
$$

When the coefficients $\alpha, \beta, \gamma$ are constant, then (1) has a steady-state solitary wave solution

$$
u(x, t)=\frac{A}{1+B \cosh K(x-V t)},
$$

where the parameters $A, B, V$ and $K$ are given by

$$
V=\alpha A=\gamma K^{2}, \quad B^{2}=1+\frac{\beta \gamma K^{2}}{\alpha^{2}} .
$$

The solitary wave solution (1) is characterised by a single parameter $B$. The wave amplitude is

$$
a=\frac{A}{1+B} .
$$

When $\beta \gamma<0$, then (1) has a single family of solitary waves such that $0<B<1$. When the amplitude is small, (1) transform into the $\mathrm{KdV}$-type solitary waves $(B \rightarrow 1)$. However, when $B \rightarrow 0$, (1) describes the so-called "thick" solitary wave (also known as "table-top" soliton ) (refer to Figure 1). On the other hand, (1) has two families of solitary waves, both with $B^{2} \rightarrow 1$ when $\beta \gamma>0$. The first has $1<B<\infty$ and ranges from small KdV-type solitary waves when $B \rightarrow 1$, to large wave with a "sech" profile as $B \rightarrow \infty$. The other family of solitary waves has the opposite polarity with $-\infty<B<-1$ and ranges from large waves with a "sech" profile to a limiting algebraic solitary wave of amplitude $-2 \alpha / \beta$ (refer to Figure 1) [9], [10].

In real world problems, internal waves are often propagating through a nonuniform environment. These internal waves have been observed in coastal regions near Malayisa, e.g. Andaman Sea [13], [14], Sulu Sea [15] and South China Sea [16] - [19]. In Sect. 2, we shall describe our problem formulation. Then in Sect. 3, we shall explain our numerical method used to solve our mathematical model. Then in Sect. 4, we shall present our numerical simulations.

\section{Problem formulation}

Internal waves in two-layer fluids is a common phenomenon near coastal areas. In order to reduce the impact from the waves, different types of coastal structures have been constructed near coastal area. The interaction between the waves in two-layer fluids and coastal structures have been studied by many researchers $[20]-[25]$. 

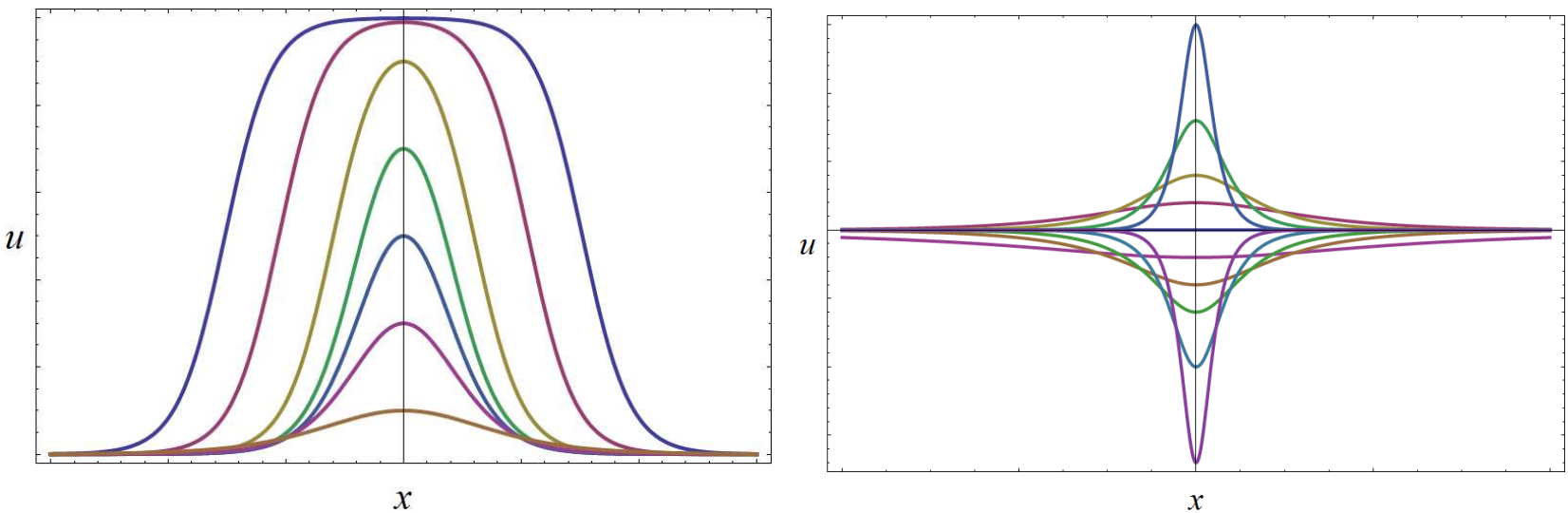

Figure 1: The Shape of Solitary Wave when Left: $\alpha>0, \beta<0, \gamma>0$, Right: $\alpha>0, \beta>0, \gamma>0$

In this paper, we consider the propagation of internal solitary waves in a two-layer fluid flow over a varying topography region. Hence, the appropriate mathematical model for this problem is the variable coefficient extended Korteweg-de Vries (veKdV) equation [see the review by Grimshaw [4], Grimshaw et al. [9], [10]]

$$
A_{t}+c A_{x}-\frac{c Q_{x}}{Q} A+\mu(x) A A_{x}+\mu_{1}(x) A^{2} A_{x}+\delta(x) A_{x x x}=0
$$

Here, $A(x, t)$ is the amplitude of the wave, and $x, t$ are the spatial and temporal variables respectively. The coefficient $c(x)$ is the relevant linear long wave speed and $Q(x)$ is the linear modification factor, defined so that $Q^{-2} A^{2}$ is the wave action flux for linear long waves. The coefficients of the nonlinear and dispersive terms, i.e. $\mu(x), \mu_{1}(x)$ and $\delta(x)$ are determined by the properties of the basic state of the fluid. All these coefficients are slowly-varying functions of $x$.

Let the densities of the fluid in the upper layer and lower layer be constants denoted by $\rho_{1}$ and $\rho_{2}$ respectively. Also, consider $H_{1}$ is the depth of the fluid in the upper layer and $H_{2}=H-H_{1}$ is the depth of the fluid in the lower layer where $H$ is the total depth. Hence, all the coefficients in (2) are given by

$$
\begin{aligned}
\mu & =\frac{3 c\left(\rho_{2} H_{1}^{2}-\rho_{1} H_{2}^{2}\right)}{2 H_{1} H_{2}\left(\rho_{2} H_{1}+\rho_{1} H_{2}\right)}, \\
\delta & =\frac{c H_{1} H_{2}\left(\rho_{2} H_{2}+\rho_{1} H_{1}\right)}{6\left(\rho_{2} H_{1}+\rho_{1} H_{2}\right)}, \\
\mu_{1} & =-\frac{3 c}{8 H_{1}^{2} H_{2}^{2}\left(\rho_{1} H_{2}+\rho_{2} H_{1}\right)^{2}}\left[\left(\rho_{1} H_{2}^{2}-\rho_{2} H_{1}^{2}\right)^{2}+8 \rho_{1} \rho_{2} H_{1} H_{2}\left(H_{1}+H_{2}\right)^{2}\right],
\end{aligned}
$$

where

$$
c^{2}=\frac{g\left(\rho_{2}-\rho_{1}\right) H_{1} H_{2}}{\rho_{1} H_{2}+\rho_{1} H_{2}}, \quad \text { and } \quad Q^{2}=\frac{1}{2 g\left(\rho_{2}-\rho_{1}\right) c} .
$$

By following the usual oceanic condition, we let $\rho_{2}>\rho_{1}$ and $\rho_{2}-\rho_{1} \ll \rho_{2}$. When the interface is closer to the free surface than the bottom surface, i.e. when $H_{1}<H_{2}$, the nonlinear coefficient $\mu$ is negative and is positive in the opposite case. 


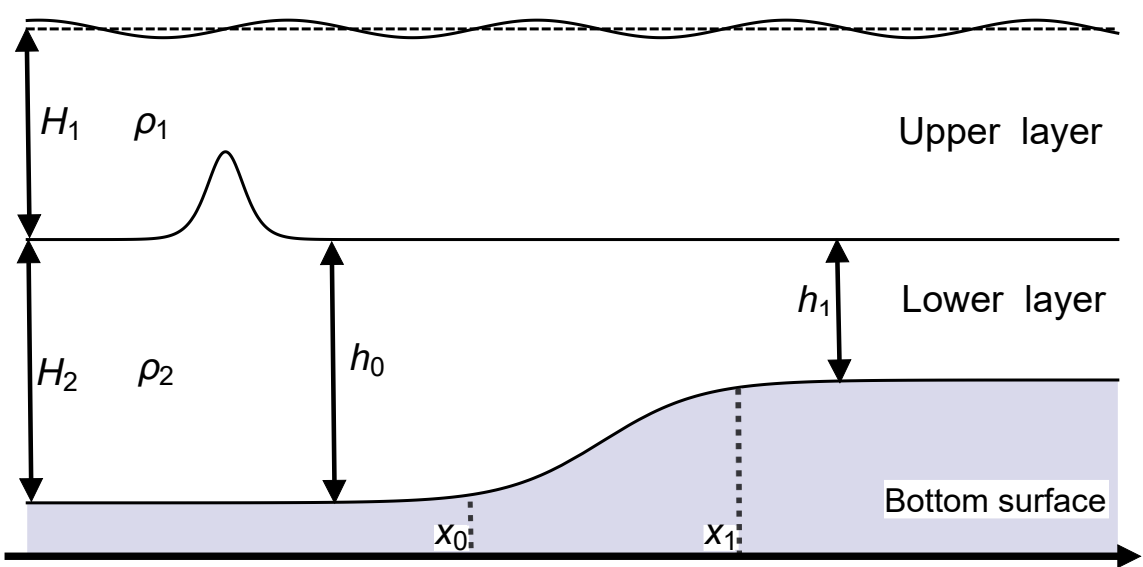

Figure 2: Schematic Illustration for the Internal Solitary WavePropagation over a Decreasing Depth Region

We shall suppose that the depth of the lower layer of the fluid flow varies according to

$$
H_{2}(x)=h_{0}=1 \text { for } x<x_{0}, \quad \text { and } \quad H_{2}(x)=h_{1} \text { for } x>x_{1} \text {, }
$$

where $h_{0}, h_{1}$ are constants and varies monotonically in the region $x_{0} \leq x \leq x_{1}$. We assume that $x_{1}-x_{0} \gg 1$. Then we shall suppose that the initial condition for $(2)$ is imposed for $x \ll x_{0}$. Thus, initially we generate a solution of the constant coefficient eKdV equation and the aim is to see how it evolves for $x>x_{0}$. In this paper we study the propagation of an internal solitary wave over a decreasing-depth region, i.e. $h_{1}<1$. The schematic of our problem is illiustrated in Figure 2.

The first two terms in (2) are the dominant terms. Thus, we can make the transformation by introducing new variables [10]

$$
A=Q U, \quad T=\int^{x} \frac{d x}{c}, \quad X=c(T-t) .
$$

On substitution into (2) gives, to the same leading order of approximation where (2) holds

$$
U_{T}+\alpha U U_{X}+\beta U^{2} U_{X}+\lambda U_{X X X}=0,
$$

where

$$
\alpha=Q \mu, \quad \beta=Q^{2} \mu_{1}, \quad \lambda=\delta .
$$

In terms of the new variables $U(X, T)$, we will consider that $H_{1}(T)$ is a constant for all $T$ and $H_{2}(T)=1$ for $0<T<T_{0}$ and varies monotonically in the interval $T_{0}<T<T_{1}$ to $H_{2}(T)=h_{1}$ when $T>T_{1}$.

\section{$3 \quad$ Numerical Method}

In this paper, we apply the method of lines (MOL) to solve the veKdV equation (3). It involves in making an approximation to the spatial derivatives. Thus, the partial differential equation is reduced into a system of ordinary differential equations which can be solved by using time 
integrator. The MOL is a powerful method to solve partial differential equations. It has been used to solve the KdV equation [26], [27], constant coefficient eKdV equation [28], [29], forced $\mathrm{KdV}$ equation [30] and forced KdV-Burgers equation [31].

First, we discretise the spatial derivative using central finite difference formulae as follows:

$$
\begin{aligned}
& U_{X}=\frac{U_{j+1}-U_{j-1}}{2 \Delta X}, \\
& U_{X X X}=\frac{U_{j+2}-2 U_{j+1}+2 U_{j-1}-U_{j-2}}{2(\Delta X)^{3}} .
\end{aligned}
$$

Here, $j$ is the index that indicate the position along spatial axis and $\Delta X$ is the increment value of spatial axis. Therefore, the MOL approximation of the veKdV equation (3) is given by

$$
\begin{aligned}
\frac{d U_{j}}{d T}= & -\frac{\alpha}{2 \Delta X} U_{j}\left(U_{j+1}-U_{j-1}\right)-\frac{\beta}{2 \Delta X} U_{j}^{2}\left(U_{j+1}-U_{j-1}\right) \\
& -\frac{\delta}{2(\Delta X)^{3}}\left(U_{j+2}-2 U_{j+1}+2 U_{j-1}-U_{j-2}\right) \\
\equiv & f\left(U_{j}\right) .
\end{aligned}
$$

For the time integration, we will use a fourth-order Runge-Kutta method. The initial condition is taken as

$$
U(X, 0)=\frac{\alpha}{\beta} \frac{B^{2}-1}{1+B \cosh (K X)},
$$

where

$$
K=\sqrt{\frac{\alpha^{2}}{6 \beta \lambda}\left(B^{2}-1\right)} .
$$

Here we consider two values for $B$, i.e. $B=0.1$ and $B=0.001$.

\section{$4 \quad$ Numerical Results}

Here, we present the numerical for the propagation of internal solitary waves over three types of varying depth region, i.e. a slowly increasing slope, a smooth bump, and a parabolic mound [32].

\subsection{Internal Solitary Wave Propagating over a Slowly Increasing Slope}

For numerical simulations, we consider the depths for both layers of the fluid as follows

$$
\begin{aligned}
& H_{1}(T)=1.5, \quad \text { for all } T \\
& H_{2}(T)=\left\{\begin{array}{lr}
1.0 & : 0 \leq T<100 \\
-0.00006 T+1.006 & : 100 \leq T<5100 \\
0.7 & : T>5100
\end{array}\right.
\end{aligned}
$$

In Figure 3, we present the numerical simulation of a KdV-type internal solitary wave $(B=0.1)$ propagating over a slowly increasing slope for the veKdV equation (3). As the internal solitary wave propagates over the slope, the solitary wave deforms adiabatically. At the same time, there 
is a non-adiabatic response in the form of an extended small-amplitude secondary structure or a shelf behind the solitary wave (refer to Figure 4). On a very long time-scale, the trailing shelf leads to the generation of an undular bore. This is due to the nonlinear effects which eventually will lead to steepening and thus the dispersive effects will be invoked. The leading wave of the trailing shelf undular bore can be interpreted as a secondary solitary wave. After the slope, the transformed internal solitary wave continues to propagate with constant velocity.

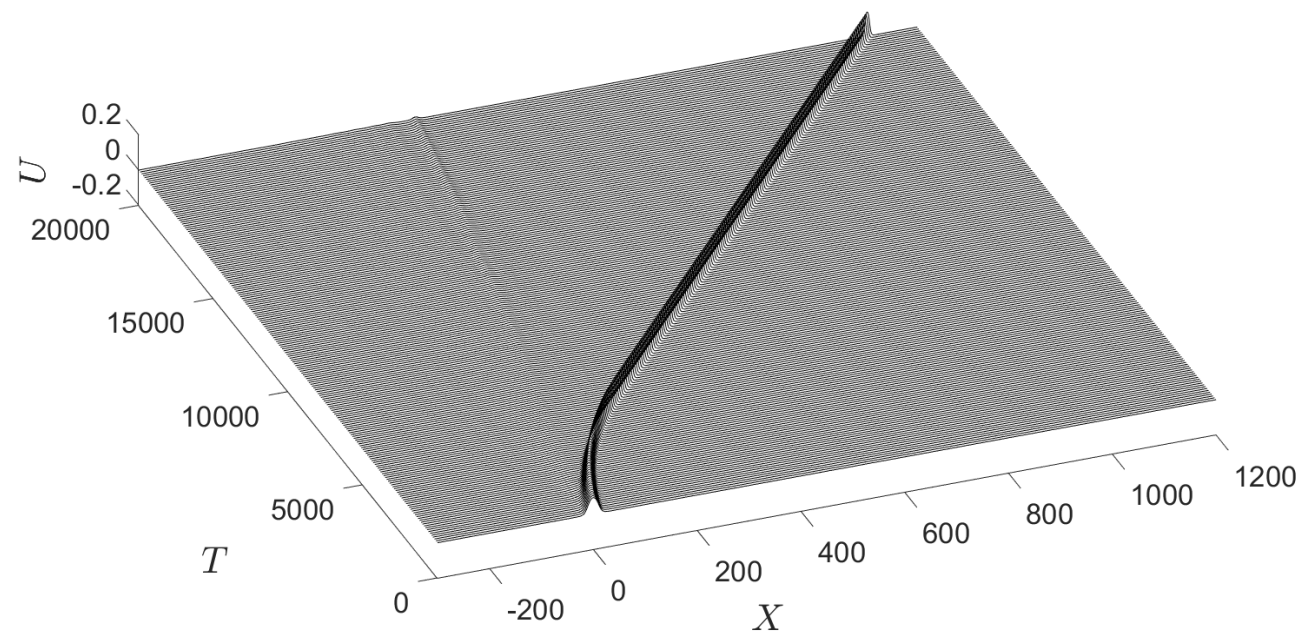

Figure 3: 3D-plot of the Propagation of a KdV-type Internal Solitary Wave Propagating over a Slowly Increasing Depth Region

Figure 5 shows the contour plot of the propagation of the internal solitary wave. It clearly shows the generation of the secondary solitary wave behind the transformed internal solitary wave.

Figure 6 shows the amplitude variation of the internal solitary wave as it propagates over the varying depth region. The amplitude of the internal solitary wave increases as it propagates over the increasing slope. After the slope, its amplitude remains constant.

When $B=0.001$, we have an initial table-top solitary wave before it enters the increasing slope region. The limiting amplitude for the table-top solitary wave before the slope is given by

$$
a_{0}=\frac{-\alpha}{\beta} \approx 0.1033 .
$$

Figure 7 presents the numerical simulation of an internal table-top solitary wave propagating over a slowly increasing slope for the veKdV equation (3). Similar to the previous case where $B=0.1$, the table-top solitary wave deforms adiabatically as it evolves on the increasing slope region. The non-adiabatic response to the interaction of the solitary wave with the varying depth region is the generation of a trailing shelf. However in this case, the mass of the shelf generated is bigger compared to previous case. As such, a wavetrain is formed behind the transformed table-top solitary wave instead of a secondary solitary wave at larger time. This is clearly shown in the 2D-plots and the contour plot (refer to Figures 8 and 9). After the slope, the initial table-top solitary wave is transformed into another table-top solitary wave with different limiting amplitude and travels with constant velocity. 

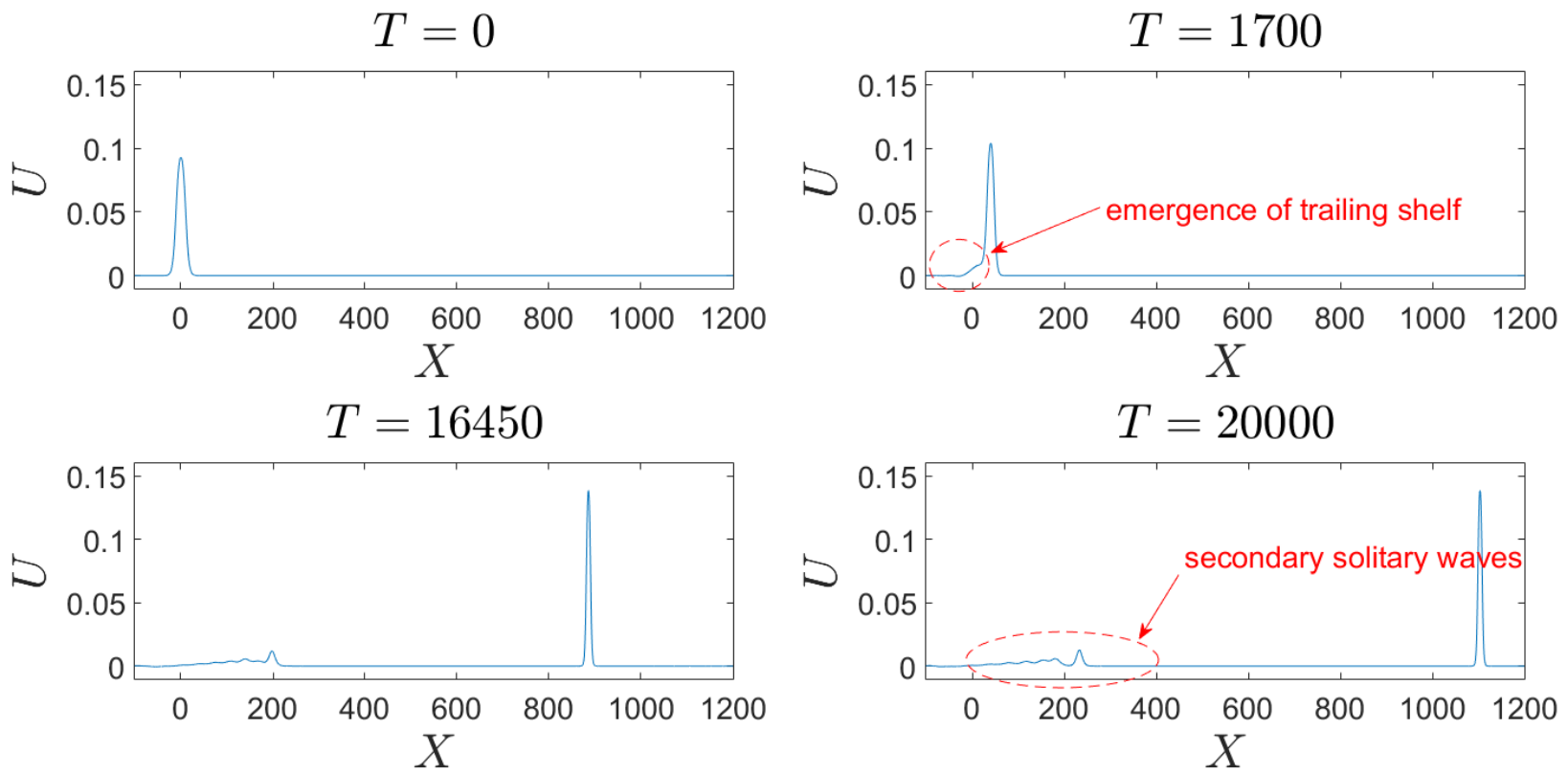

Figure 4: 2D Plots of a KdV-type Internal Solitary Wave Propagating over a Slowly Increasing Depth Region

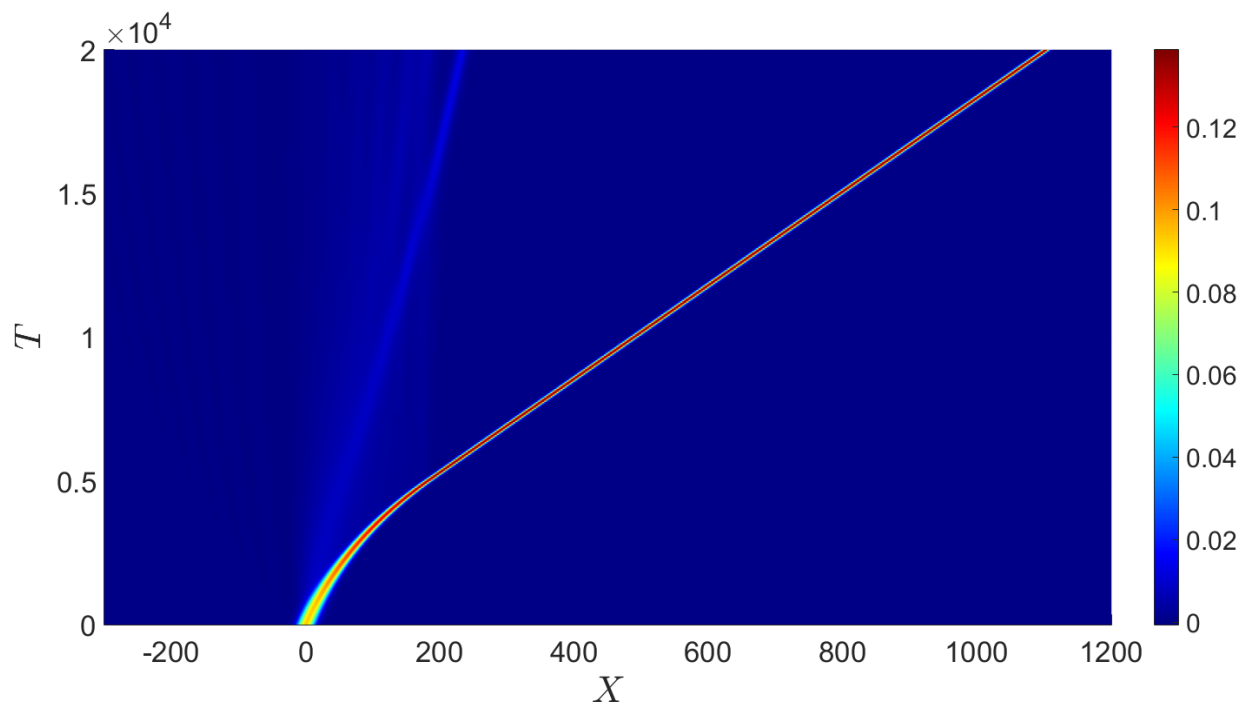

Figure 5: Contour Plot of a KdV-type Internal Solitary Wave Propagating over a Slowly Increasing Depth Region 


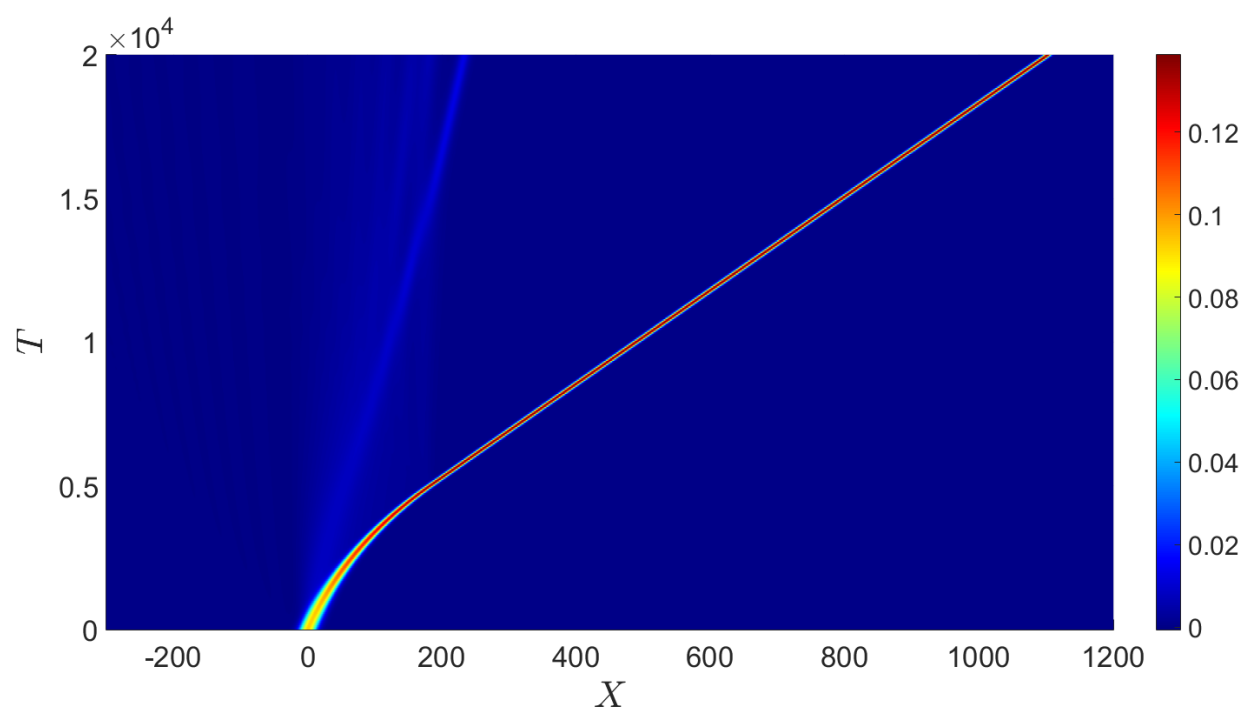

Figure 6: Amplitude Variation of Adiabatically Changing for Table-top Solitary Wave and KdV-type Wave

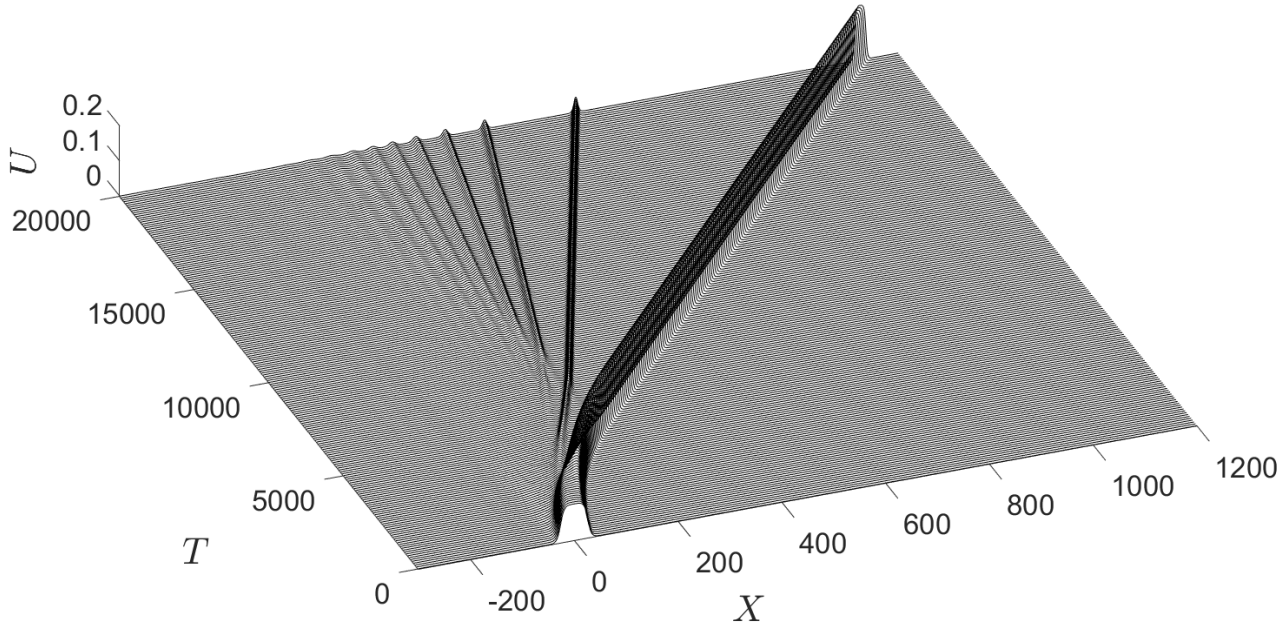

Figure 7: 3D-plot of the Propagation of a Table-top Solitary Wave Propagating over a Slowly Increasing Depth Region 

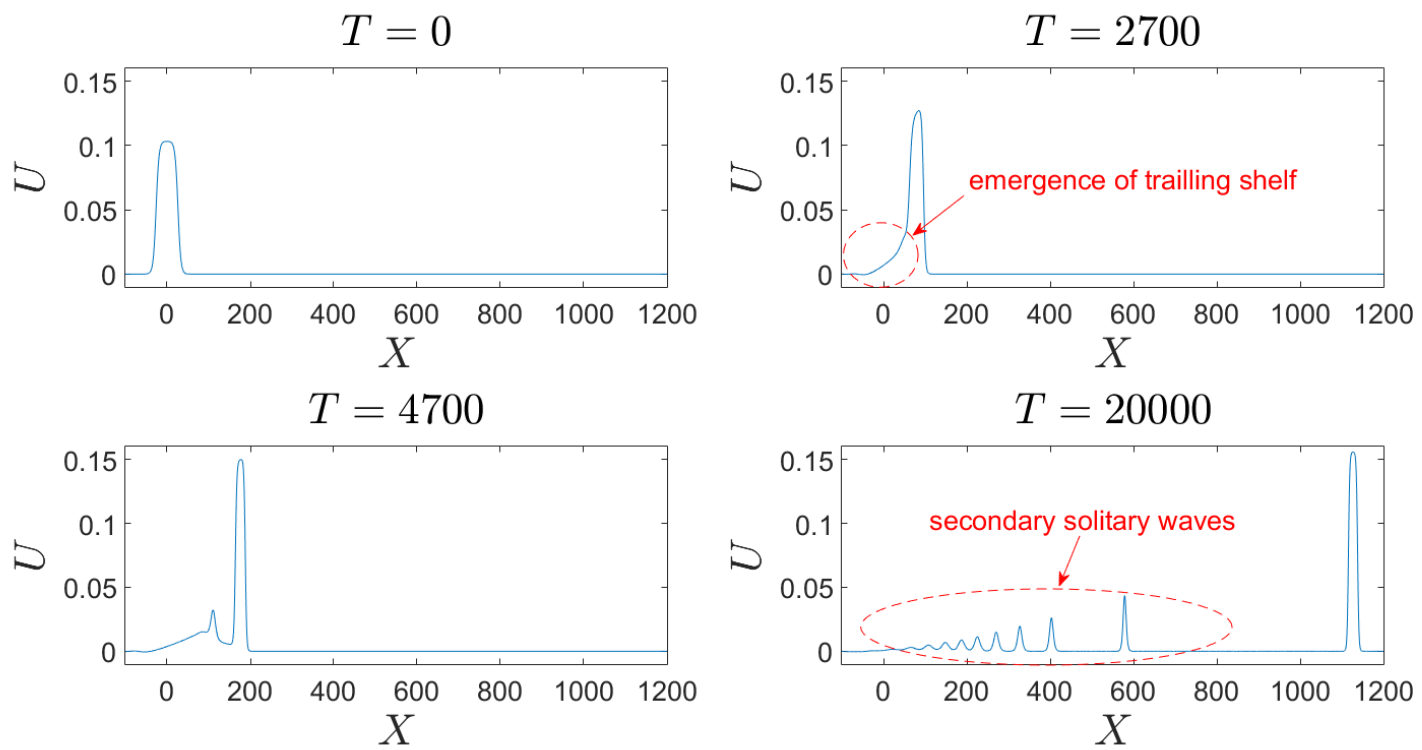

Figure 8: 2D Plots of a Table-top Solitary Wave Propagating over a Slowly Increasing Depth Region

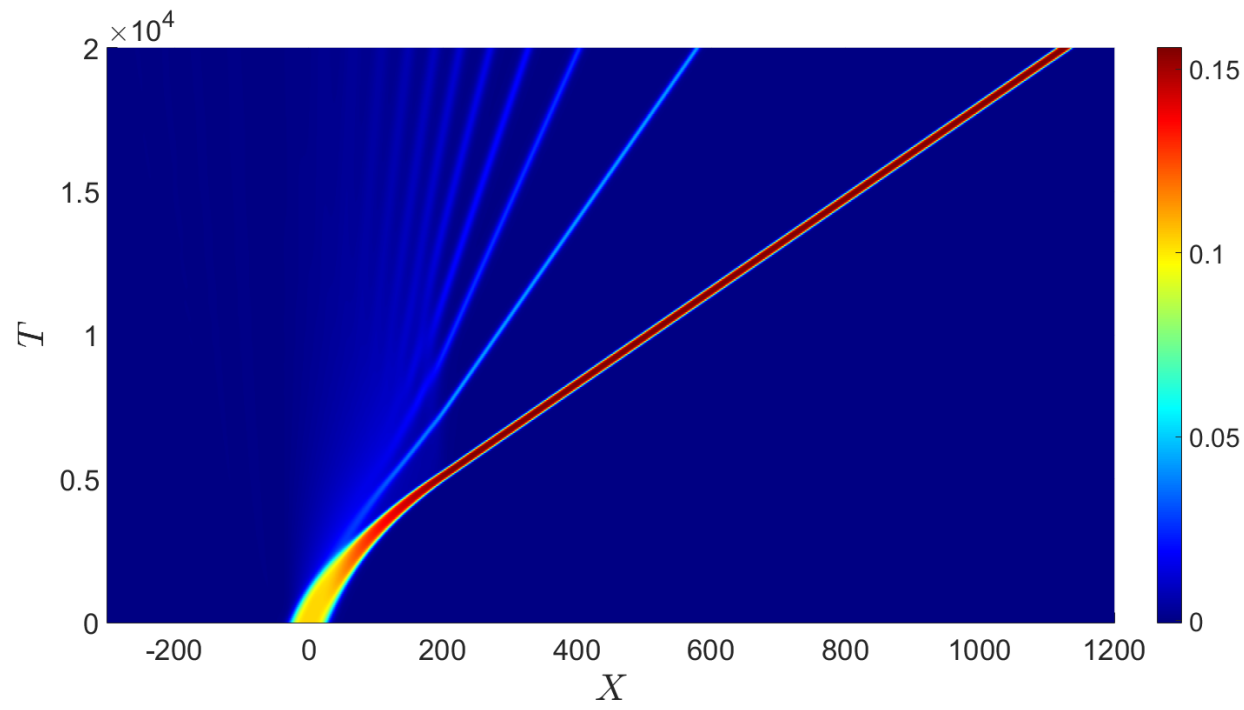

Figure 9: Contour Plot of a Table-top Solitary Wave Propagating over a Slowly Increasing Depth Region

The new limiting amplitude after the slope is given by

$$
a_{1} \approx 0.1560
$$

This is shown clearly in Figure 6. Therefore, the transformed solitary wave after the slope is a table-top solitary wave as well. Again, the amplitude of the solitary wave increases as it evolves on the slope. Once it reaches another constant depth region, its amplitude remains unchanged. 


\subsection{Internal Solitary Wave Propagating over a Smooth Bump}

In this subsection, we consider internal solitary wave propagating over a smooth bump. The depth profile for both layers is given by:

$$
\begin{aligned}
& H_{1}(T)=1.5, \quad \text { for all } T, \\
& H_{2}(T)=\left\{\begin{array}{lr}
1.0 & : 0 \leq T<100 \\
-0.00006 T+1.006 & : 100 \leq T<5100 \\
0.7 & : 5100 \leq T<5600 \\
0.00006 T+0.364 & : 5600 \leq T<10600 \\
1.0 & : T>10600
\end{array}\right.
\end{aligned}
$$

As mentioned in the previous subsection, the internal wave deform adiabatically as it propagates over the slowly increasing slope region and producing a trailing shelf at the same time, which would then generate secondary solitary waves as time increases. When it propagates over the slowly decreasing slope region, similarly the internal solitary wave deforms adiabatically. However a trailing shelf of negative polarity is generated in this case. The results are shown in Figures $10-11$ for both $B=0.1$ and $B=0.001$. The trailing shelf of negative polarity acts as an pedestal for the secondary solitary wavetrain to climb as it enter the new constant depth region after the bump. Again, we observed a train of secondary solitary waves is generated behind the transformed solitary waves after the bump. The 3D-plots are shown in Figures 12 and 13 .
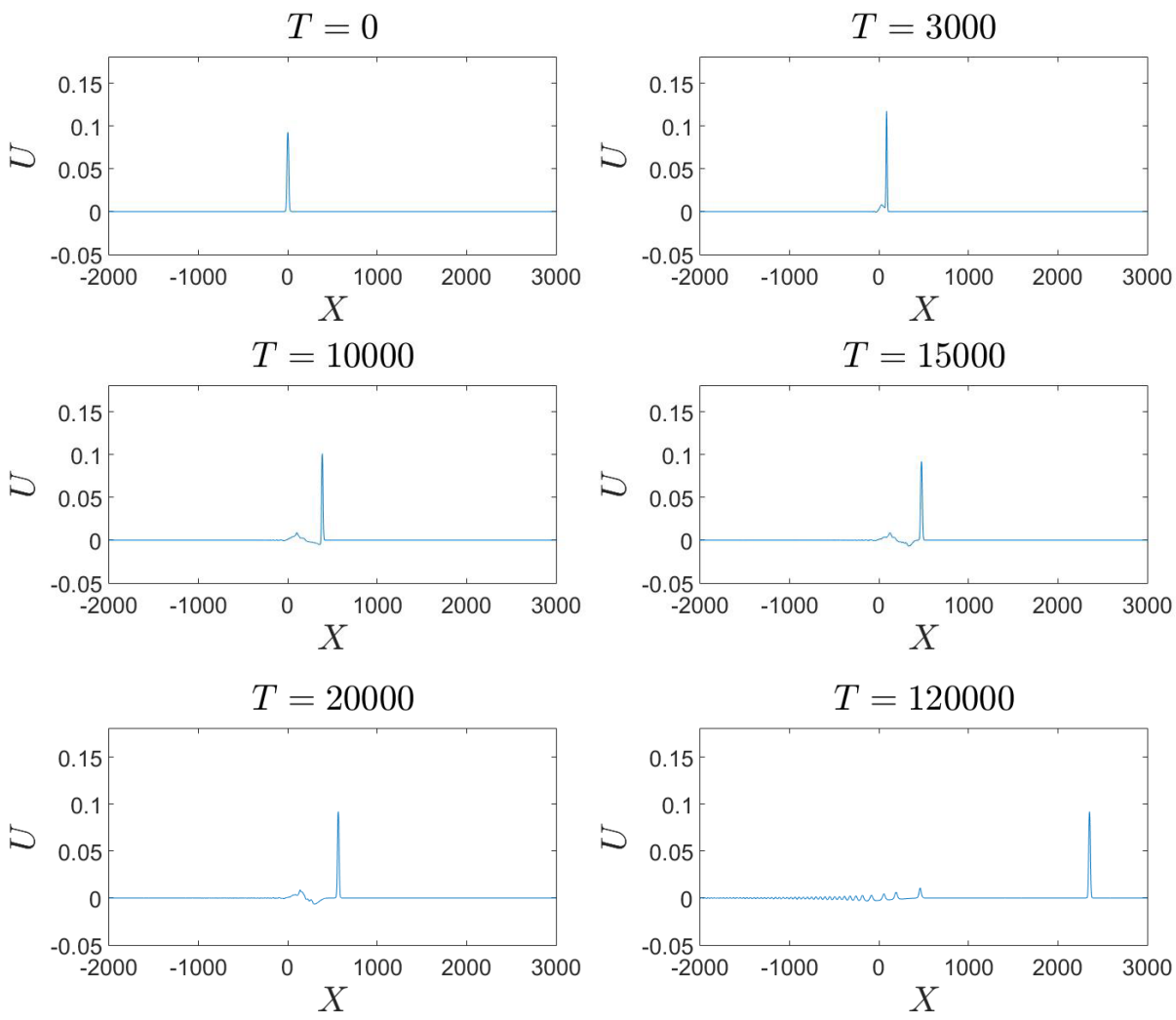

Figure 10: 2D Plots of a KdV-type Internal Solitary Wave Propagating over a Smooth Bump 

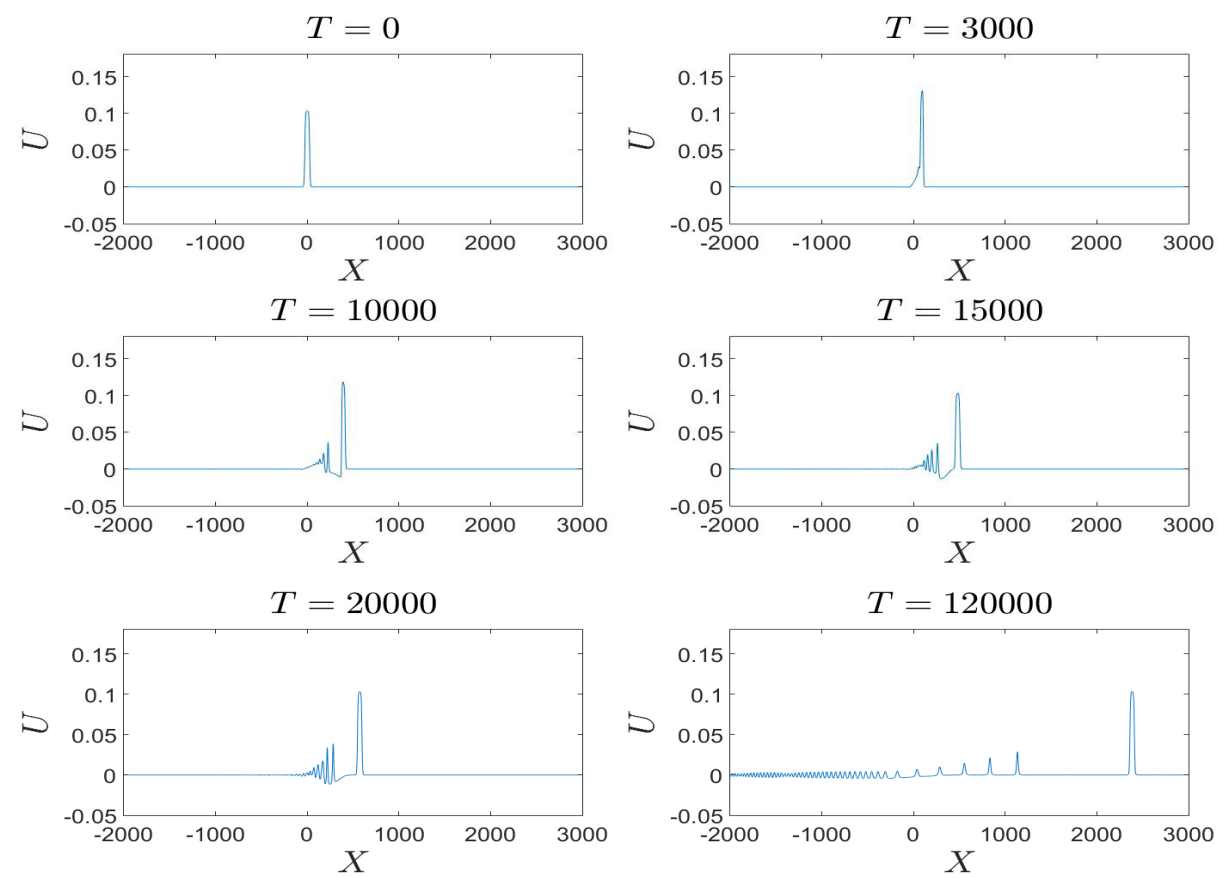

Figure 11: 2D Plots of a Table-top Solitary Wave Propagating over a Smooth Bump

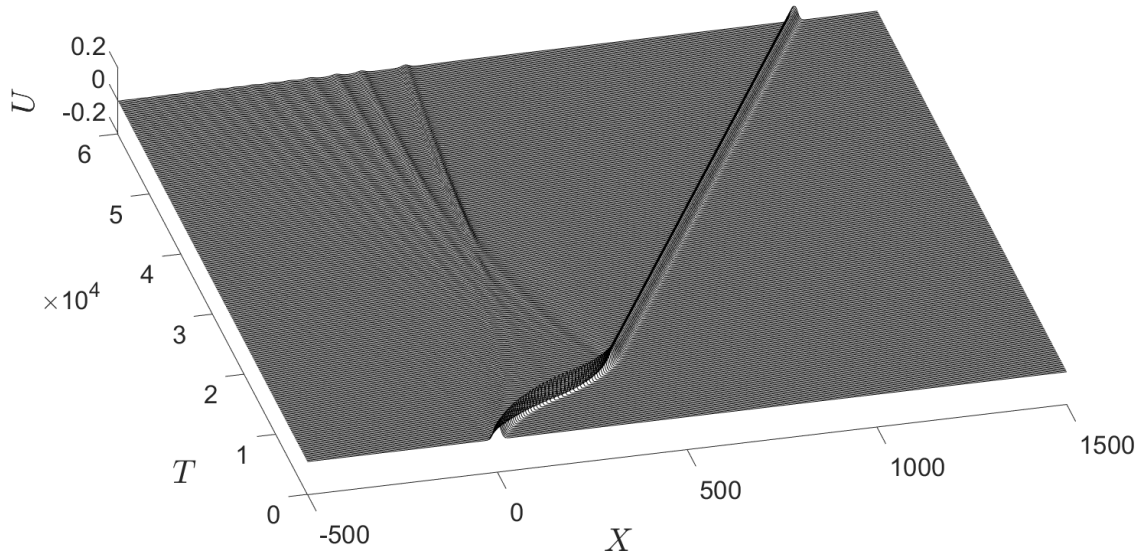

Figure 12: 3D-plot of the Propagation of a KdV-type Internal Solitary Wave Propagating over a Smooth Bump 


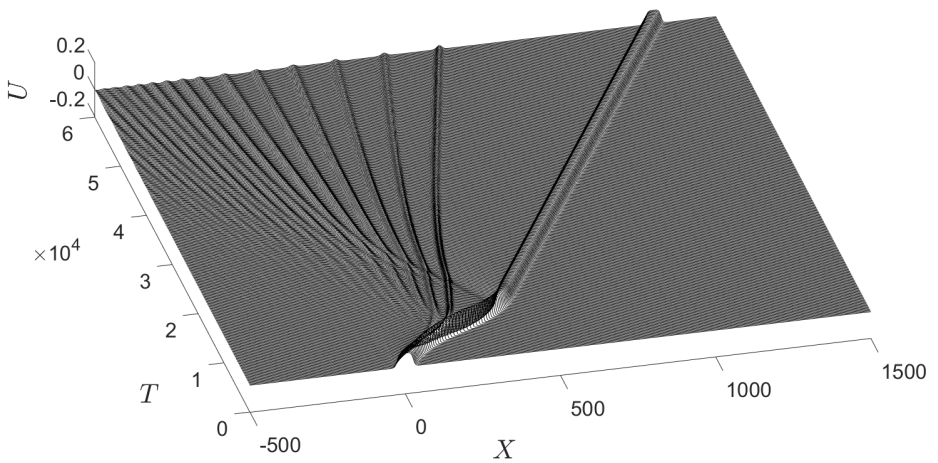

Figure 13: 3D-plot of the Propagation of a Table-top Solitary Wave Propagating over a Smooth Bump

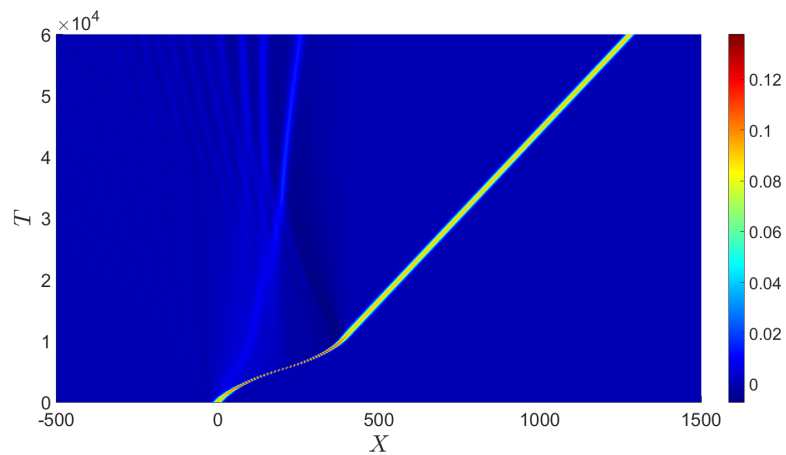

Figure 14: Contour Plot of a KdV-type Internal Solitary Wave Propagating over a Smooth Bump

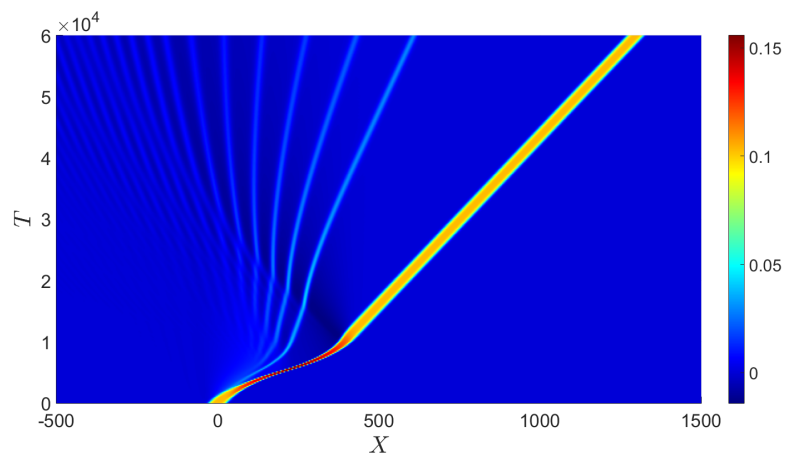

Figure 15: Contour Plot of a Table-top Solitary Wave Propagating over a Smooth Bump

The generation of trailing shelf of negative polarity is clearer in the contour plots (see Figures 14 and 15). The amplitude of the internal solitary wave decreases when it propagates over the decreasing slope region of the bump. This is shown in Figures 16. The amplitude of the internal solitary wave increases while it is on the increasing slope and reduces when it enters the decreasing slope region. Its amplitude remains constant after the slope. The amplitude of the transformed solitary waves after the slope is slightly less than the amplitude of the initial internal solitary waves. 


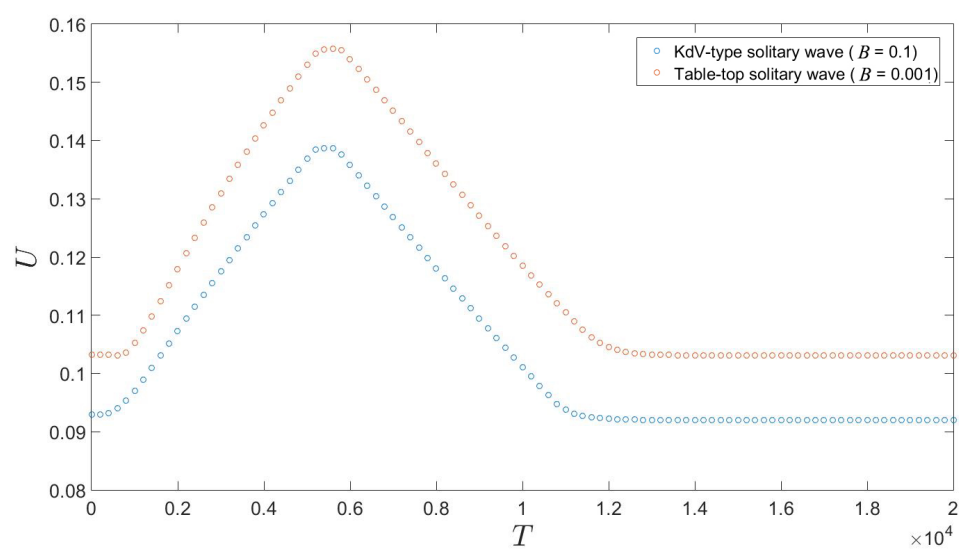

Figure 16: Amplitude Variation of Internal Solitary Waves Propagating over a Smooth Bump

\subsection{Internal Solitary Wave Propagating Over a Parabolic Mound}

In this subsection, we consider the case where internal solitary wave is propagating over a parabolic mound, which is described by

$$
\begin{aligned}
& H_{1}(T)=1.5, \quad \text { for all } T \\
& H_{2}(T)=\left\{\begin{array}{lr}
1.0 & : 0 \leq T<300 \\
(1 / 27075000) T^{2}+(-21 / 90250) T+(385 / 361) & : 300 \leq T<6000 \\
1.0 & : T>6000
\end{array}\right.
\end{aligned}
$$

In this case, the numerical results are similar to the propagation of internal waves over a smooth bump. When the internal solitary wave propagates over an increasing slope of the mound, the internal solitary wave deforms adiabatically on the increasing slope region and generating a trailing shelf of positive polarity behind the solitary wave. When the internal solitary wave enters the decreasing slope region, again it deforms adiabatically and generating a trailing shelf of negative polarity. The results for both KdV-type and table-top solitary waves propagating over the parabolic mound are shown in Figures $17-20$.

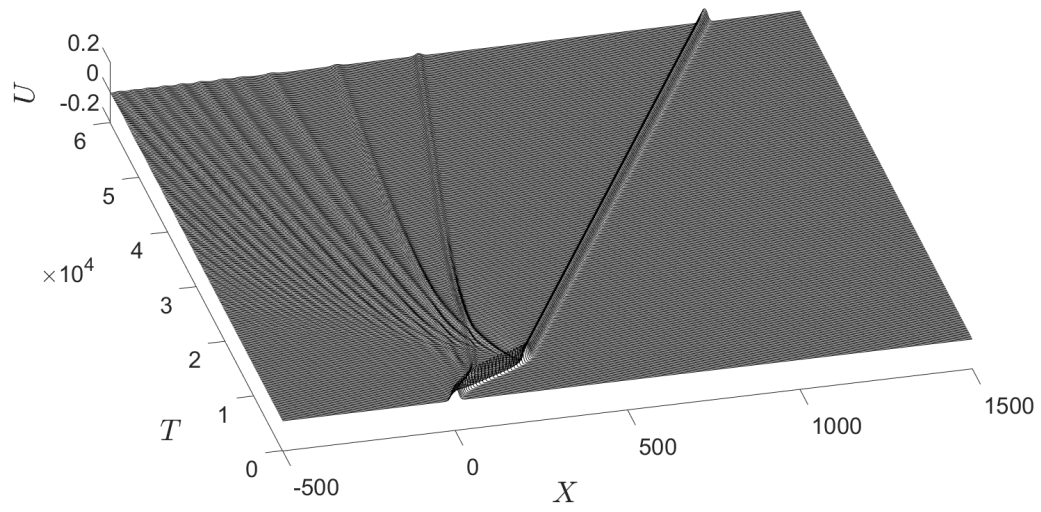

Figure 17: 3D-plot of a KdV-type Internal Solitary Wave Propagating over a Parabolic Mound 

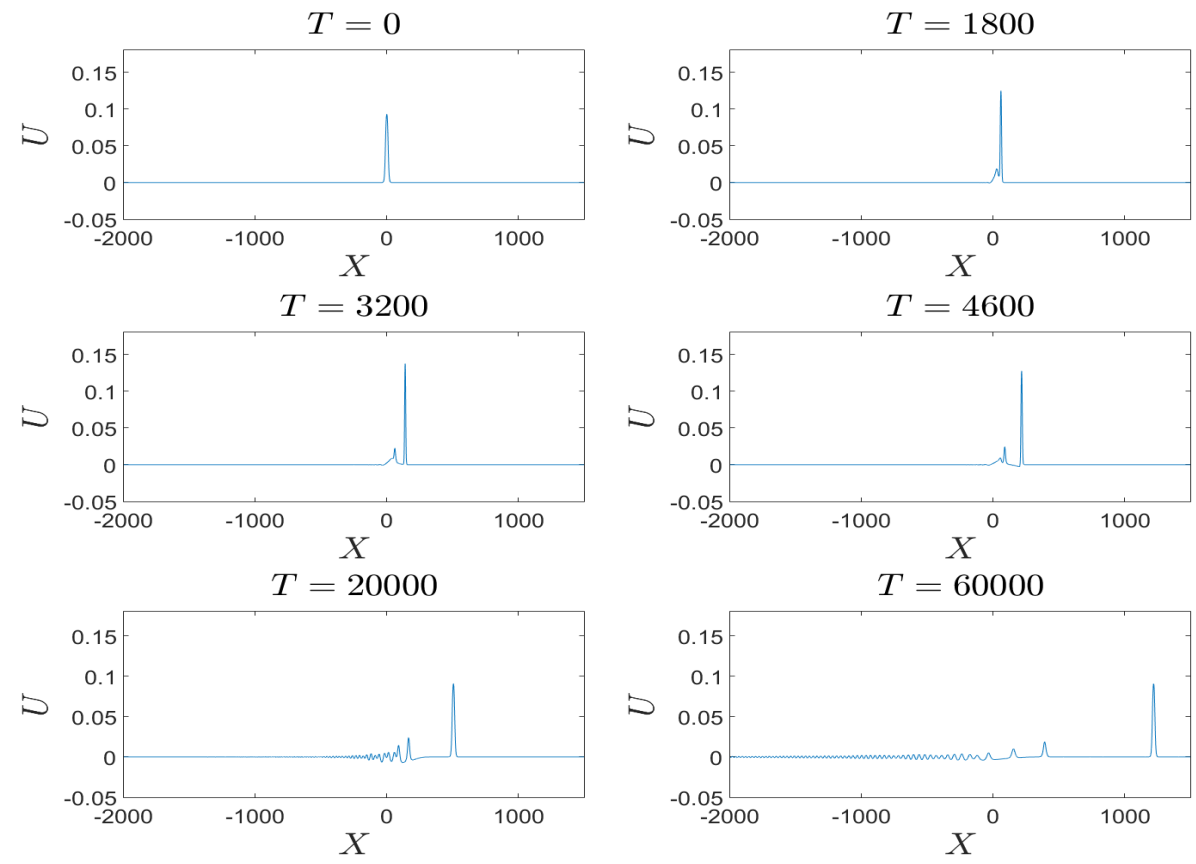

Figure 18: 2D Plots of a KdV-type Internal Solitary Wave Propagating over a Parabolic Mound

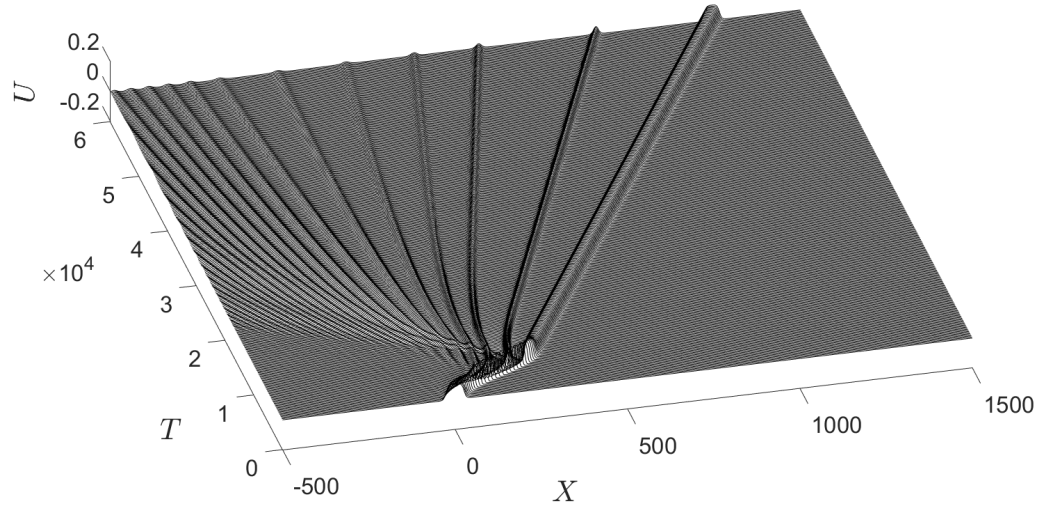

Figure 19: 3D-plot of a Table-top Solitary Wave Propagating over a Parabolic Mound 

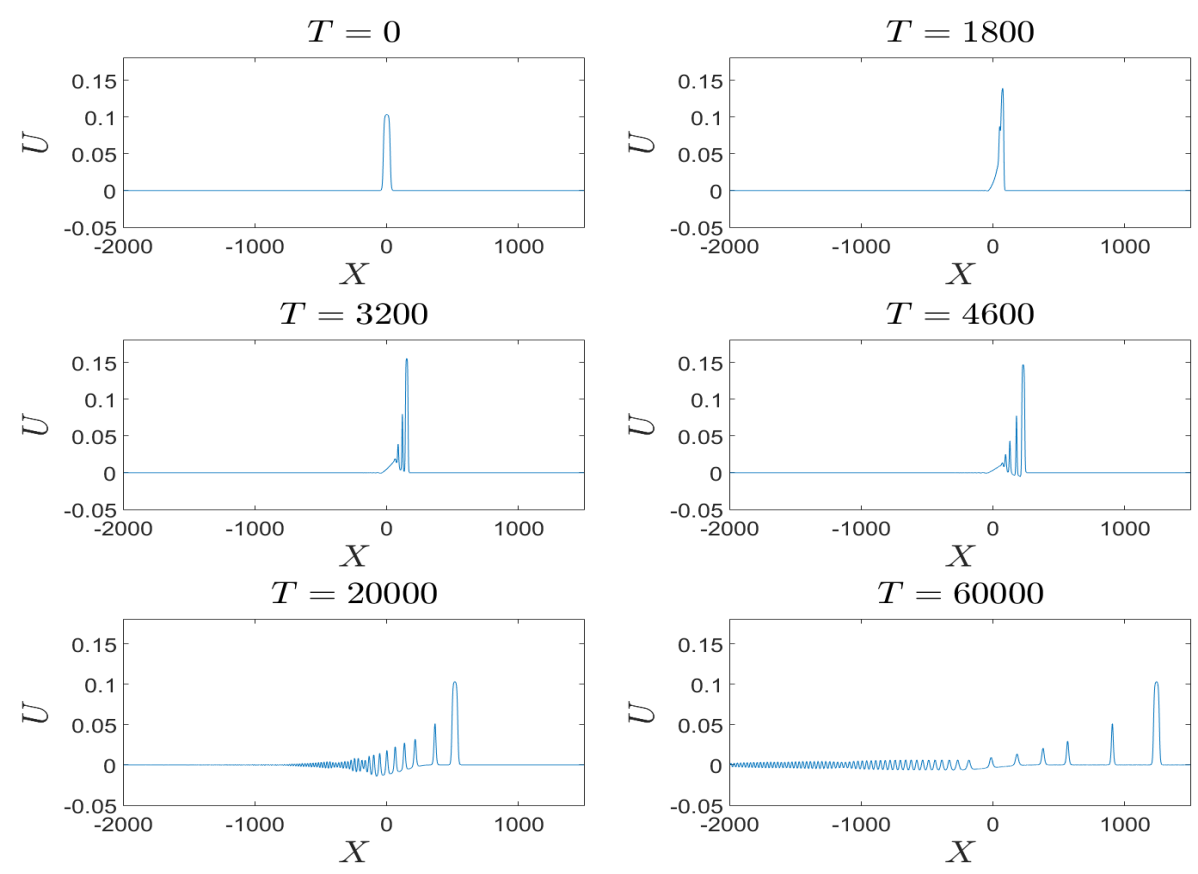

Figure 20: 2D Plots of a Table-top Solitary Wave Propagating over a Parabolic Mound

The contour plots of the KdV-type solitary wave and table-top solitary wave propagating over a parabolic mound is shown in Figures 21 and 22. Similar to the smooth bump, a train of secondary solitary waves is generated and propagating behind the transformed internal wave after the parabolic mound.

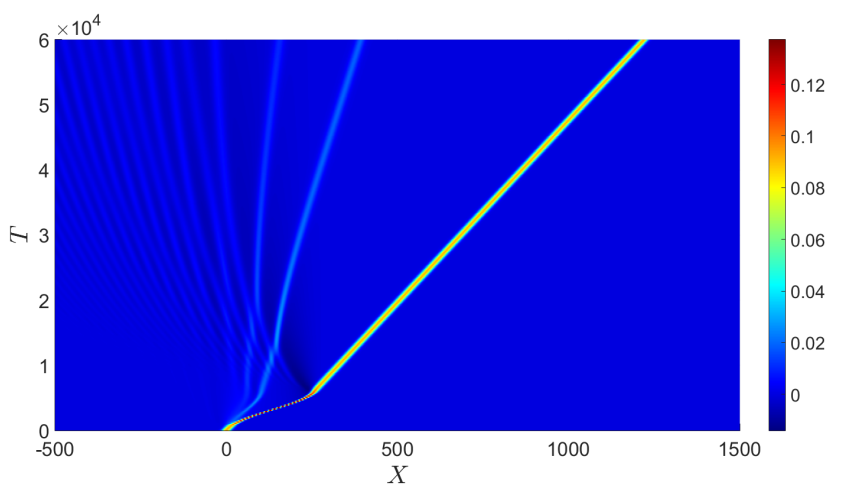

Figure 21: Contour Plot of a KdV-type Internal Solitary Wave Propagating over a Parabolic Mound 


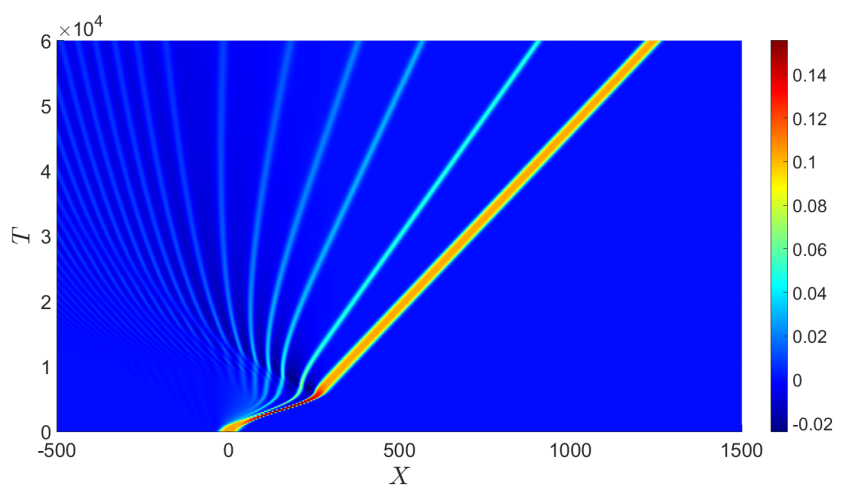

Figure 22: Contour Plot of a Table-top Solitary Wave Propagating over a Parabolic Mound

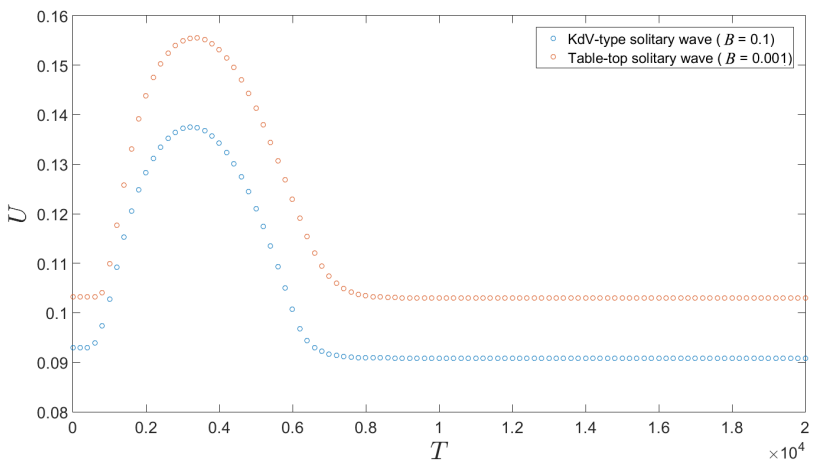

Figure 23: Amplitude Variation of Internal Solitary Waves Propagating over a Parabolic Mound

The amplitude variation of the internal solitary wave as it propagates over a parabolic mound for both cases are shown in Figure 23. Similar to the previous case of internal solitary wave propagating over a smooth bump, the amplitude of wave increases when it propagates over the increasing slope region and decreases after it propagates over the decreasing slope region.

\section{Conclusion}

In this paper, we have looked into internal solitary wave propagation over a variable topography. We have considered the configuration where the water depth decreases slowly. As the result of the interaction of increasing slope region on the evolution of the internal solitary wave, a trailing shelf is formed behind the solitary wave. The trailing shelf will decompose into secondary solitons or a wavetrain depending on the mass of the shelf. This is similar to the soliton fission scenario, albeit on a much larger temporal scale. Thus, the propagation of the solitary wave over slowly varying topography leads to a non-adiabatic response regardless of the smallness of the bottom slope variation. When the internal solitary waves propagates over the decreasing slope region of the bump and parabolic mound, the internal waves deform adiabatically. Its amplitude decreases when it evolves over the decreasing slope. The non-adiabatic response is the generation of the trailing shelf of negative polarity. 


\section{Acknowledgments}

The authors would like to thank the referees for their valuable comments and suggestions.

\section{References}

[1] Benney, D. J. Long non-linear waves in fluid flows. J. Math. Phys.. 1966. 45: 52-63.

[2] Benjamin, T. B. Internal waves of finite amplitude and permanent form. Journal of Fluid Mechanics. 1966. 25: 241-270.

[3] Apel, J., Ostrovsky, L. A., Stepanyants, Y. A., and Lynch, J. F. Internal solitons in the ocean and their effect on underwater sound, J. Acoust. Soc. Am.. 2007. 121: 695-722.

[4] Grimshaw, R. Internal solitary waves. In Grimshaw, R. (Ed.). Environmental Stratified Flows. Boston: Kluwer. 2001. 1- 28.

[5] Helfrich, K. R. and Melville,W. K. Long nonlinear internal waves, Annu. Rev. Fluid Mech.. 2006. 38: 395-425.

[6] Holloway, P., Pelinovsky, E., and Talipova, T. Internal tide transformation and oceanic internal solitary waves. In Grimshaw, R. (Ed.). Environmental Stratified Flows. Boston: Kluwer. 2001. 29-60.

[7] Grimshaw, R., Pelinovsky, E., Talipova, T. and Kurkin, A., Simulation of the Transformation of Internal Solitary Waves on Oceanic Shelves. Journal of Physical Oceanography. 2004. 34: 2774-2791.

[8] Grimshaw, R., Pelinovsky, E., Talipova, T. and Kurkina, O., Internal solitary waves: propagation, deformation and disintegration. Nonlin. Processes Geophys. 2010. 17: 633649.

[9] Grimshaw, R., Pelinovsky, E., and Talipova, T. Damping of large amplitude solitary waves. Wave Motion. 2003. 37: 351-364.

[10] Grimshaw, R., Pelinovsky, E., and Talipova, T. Modelling internal solitary waves in the coastal ocean. Surveys in Geophysics. 2007. 28: 273-298.

[11] Tiong, W. K., Tay, K. G., Ong, C. T. and Chiew, K. L. Numerical simulation of undular bores evolution with Chezy friction. In Proceedings of 2015 International Symposium on Mathematical Sciences and Computing Research (iSMSC), May 19-20. Ipoh, Perak. 414418

[12] Tiong, W. K., Tay, K. G. and Chiew, K. L. Simulation of undular bores evolution with damping. Dynamics of Continuous, Discrete and Impulsive Systems Series B: Applications \& Algorithms 2017. 24: 113-126.

[13] Vlasenko, V. I. and Hutter, K. Transformation and disintegration of strongly nonlinear internal waves by topography in stratified lakes. Ann. Geophys. 2002. 20: 2087-2103.

[14] Vlasenko, V. I., Stashchuk, N. M. and Hutter, K. Baroclinic Tides: Theoretical Modelling and Observational Evidence. Cambridge: Cambridge University Press. 2005.

[15] Grue, J. Generation, propagation and breaking of internal solitary waves. Chaos. 2005. 15: 037110. 
[16] Duda, T. F., Lynch, J. F., Irish, J. D., Beardsley, R. C., Ramp, S. R., Chiu, C.-S., Tang, T. Y. and Yang, Y.-J. Internal tide and nonlinear internal wave behavior at the continental slope in the northern south China Sea. IEEE J. Oceanic Eng. 2004. 29: 1105-1130.

[17] Liu, A. K., Chang, Y. S., Hsu, M.-K. and Liang, N. K. Evolution of nonlinear internal waves in the East and South China Seas. J. Geophys. Res. 1998. 103: 7995-8008.

[18] Liu, A. K., Ramp, S. R., Zhao, Y. and Tang, T. Y. A case study of internal solitary wave propagation during ASIAEX 2001. IEEE J. Oceanic Eng. 2004. 29: 1144-1156.

[19] Orr, M. H. and Mignerey, P. C. Nonlinear internal waves in the South China Sea: Observation of the conversion of depression internal waves to elevation internal waves. J. Geophys. Res. 2003. 108: 3064.

[20] Kuznetsov, N. Trapped modes of internal waves in a channel spanned by a submerged cylinder. Journal of Fluid Mechanics. 1993. 254: 113 - 126.

[21] Linton, C. M. and Cadby, J. R. Scattering of oblique waves in a two-layer fluid. Journal of Fluid Mechanics. 2002. 461: 343 - 364.

[22] Behera, H., Mandal, S. and Sahoo, T. Oblique wave trapping by porous and flexible structures in a two-layer fluid. Physics of Fluids. 2013. 25: 112110.

[23] Behera, H. and Sahoo, T. Gravity wave interaction with porous structures in two-layer fluid. Journal of Engineering Mathematics. 2014. 87: 73 - 97.

[24] Mandal, S., Behera, H. and Sahoo, T. Oblique wave interaction with porous, flexible barriers in a two-layer fluid. Journal of Engineering Mathematics. 2016. 100: 1 - 31.

[25] Behera, H. and Ng, C.-O. Interaction between oblique waves and multiple bottom-standing flexible porous barriers near a rigid wall. Meccanica. 2018. 53: 871-885.

[26] Schiesser, W. E. The numerical method of lines: Integration of partial differential equations. San Diego, California: Academic Press. 1991

[27] Schiesser, W. E. Method of lines solution of the Korteweg-de Vries equation. Computers and Mathematics with Applications. 1994. 28: 147-154.

[28] Marchant, T. R. and Smyth, N. F. Solitary wave interaction for the extended Korteweg-de Vries equation. IMA Journal of Applied Mathematics 1996. 56: 157-176.

[29] Tiong, W. K., Tay, K. G., Ong, C. T. and Sze, S.N. Numerical Solution of the Gardner Equation. In Ahmad, A.-R., Kor, L.K., Ahmad, I. and Idurs, Z. (Eds). Proceedings of the International Conference on Computing, Mathematics and Statistics (iCMS 2015): Bridging Research Endeavors. Singapore: Springer Nature. 2017. 243-251

[30] Yazid, N. M., Tay, K. G, Choy, Y. Y., Sudin, A. M., Tiong, W. K., and Ong, C. T. Numerical solution of the forced Korteweg-de Vries equation. ARPN Journal of Engineering and Applied Sciences 2016. 11: 10757-10760.

[31] Yazid, N. M., Tay, K. G, Tiong, W. K., Choy, Y. Y., Sudin, A. M. and Ong, C. T. The method of lines solution of the Forced Korteweg-de Vries-Burgers equation. MATEMATIKA 2017. 33: 35-41.

[32] Manam, S. R. and Kaligatla, R. B. A mild-slope model for membrane-coupled gravity waves. Journal of Fluids and Structures 2012. 30: 173-187. 